\title{
Feocromocitoma-ganglioneuroma compuesto en paciente con neurofibromatosis tipo 1
}

\author{
Composite pheochromocytoma-ganglioneuroma \\ in patient with neurofibromatosis type 1
}

\author{
María Vicente Santos, Ana Herrero Ruiz, Manuel Delgado Gómez, Sylvie 0jeda Rodriguez \\ Hospital Clínico Universitario de Salamanca
}

\begin{abstract}
Resumen
La neurofibromatosis tipo 1 (NF-1) o enfermedad e Von Recklinhousen es una enfermedad multisistémica de herencia autosómica dominante que afecta principalmente a la piel y al sistema nervioso. El diagnóstico es clínico y puede confirmarse por estudio genético, pero técnicamente es complejo y no predice la aparición de complicaciones, por lo que no está indicado realizarlo de forma rutinaria. La neurofibromatosis tipo 1 se asocia a distintas enfermedades endocrinológicas, siendo una de ellas el feocromocitoma. Los feocromocitomas compuestos son tumores raros que también se han relacionado con este síndrome. Asocian feocromocitomas con tumores con un mismo origen embrionario, siendo los que asocian ganglioneuromas los más frecuentes. La prevalencia de feocromocitoma y ganglioneuroma puede estar aumentada en pacientes con NF-1 y esta asociación se ha relacionado con tumores más agresivos, por lo que en el presente artículo se insiste en la importancia de la evaluación de estos pacientes para evitar posibles complicaciones relacionadas con el tumor si no se hace un diagnóstico precoz.
\end{abstract}

Presentamos el caso de un feocromocitoma compuesto con ganglioneuroma en una paciente asintomática afecta de NF-1 y se revisan los aspectos más relevantes de este tipo de tumores

Palabras clave: Feocromocitoma compuesto. Ganglioneuroma. Neurofibromatosis tipo 1

\section{Introducción}

Los feocromocitomas compuestos son tumores muy raros, con menos de 50 casos descritos en toda la literatura ${ }^{1}$. Asocian feocromocitomas con tumores de un mismo origen embrionario, incluyéndose en los casos descritos la presencia de ganglioneuromas ${ }^{2,3}$, ganglioneuroblastomas ${ }^{4}$, neuroblastomas, schawannomas y carcinomas neuroendocrinos ${ }^{5}$. Con frecuencia, aparecen asociados a enfermedades familiares, como NF-1 y neoplasia endocrina múltiple MEN-2A $\mathrm{A}^{3,4,6}$.

Presentamos el caso de un feocromocitoma compuesto en una paciente afecta de NF-1.

\section{Caso clínico}

Mujer de 28 años, con diagnóstico de NF-1 confirmada genéticamente (mutación c.2446c>Tp.R816 X, exón 16 gen NF1 en heterocigosis). Entre los antecedentes personales destacaba un bocio multinodular simple con anatomía patológica de hiperplasia nodular, anemia ferropénica en tratamiento con hierro oral y escoliosis en seguimiento por traumatología. Había sido intervenida por dermatología con extirpación de neurofibromas múltiples en espalda. Entre los antecedentes familiares, existía la sospecha de NF-1 en su madre y en su abuela (no habían sido estudiadas). Es remitida

\begin{abstract}
Neurofibromatosis type 1 (NF-1) or Von Recklinhousen's disease is a multisystem disease of autosomal dominant inheritance that primarily affects the skin and nervous system. Diagnosis is clinical and can be confirmed by genetic testing, but technically complex and does not predict the occurrence of complications, so performing it routinely is not indicated. Neurofibromatosis type 1 is associated with various endocrine diseases, one of which pheochromocytoma. The compounds pheochromocytomas are rare tumours which have also been associated with this syndrome. Pheochromocytomas are associated with tumours with the same embryonic origin, the ganglioneuromas being the most frequent. The prevalence of pheochromocytoma and ganglioneuroma may be increased in patients with NF-1 and this has been associated with more aggressive tumours, so this article stresses the importance of evaluating these patients to avoid complications related to the tumour if there was not an early diagnosis.

We present the case of a pheochromocytoma compound with ganglioneuroma in an asymptomatic patient affected from NF-1 and the most relevant aspects of this tumour are reviewed.
\end{abstract}

Key words: Composite pheochromocytoma. Ganglioneuroma. Neurofibromatosis type 1

a nuestro servicio para estudio de masa adrenal descubierta en un TAC abdominal solicitado desde el servicio de dermatología. En la anamnesis, refería la presencia de manchas café con leche desde la infancia y la aparición de otras lesiones, blandas al tacto, desde los 14 años, distribuidas de forma generalizada. Estaba asintomática, sin clínica de patología adrenal. En la exploración destacaba una tensión arterial normal

(110/70 mmHg) y la presencia de manchas café con leche generalizadas, de predominio en tronco y extremidades, de diferentes tamaños y efélides de predominio en axilas.

Junto a estas lesiones tenía otras papulosas y nodulares blandas, también distribuidas de forma generalizada, compatibles con neurofibromas. Presentaba bocio II/III a expensas del lóbulo tiroideo derecho y escoliosis leve, siendo el resto de la exploración normal. En el TAC abdominal se observaba una gran masa suprarrenal izquierda de 4,8 × 4,4 × 2,9 cm de diámetro, de contornos nítidos, con captación heterogénea del contraste por la presencia de necrosis (Figura 1).

La analítica general y el estudio hormonal para descartar el carácter funcionante de la masa, que incluía cortisol y ACTH basales, cortisol libre en orina de 24 horas y cortisol tras supresión nocturna con $1 \mathrm{mg}$ de dexametasona, DHEA-S, aldosterona y renina, fueron normales. La determinación de catecolaminas y metanefrinas fraccionadas en orina y de su metabolito el ácido vanilmandélico 
Figura 1. TAC abdominal: masa en suprarrenal izquierda de 4,8×4,4×2,9 cm de diámetro, de contornos nítidos, con captación heterogénea del contraste por presencia de necrosis

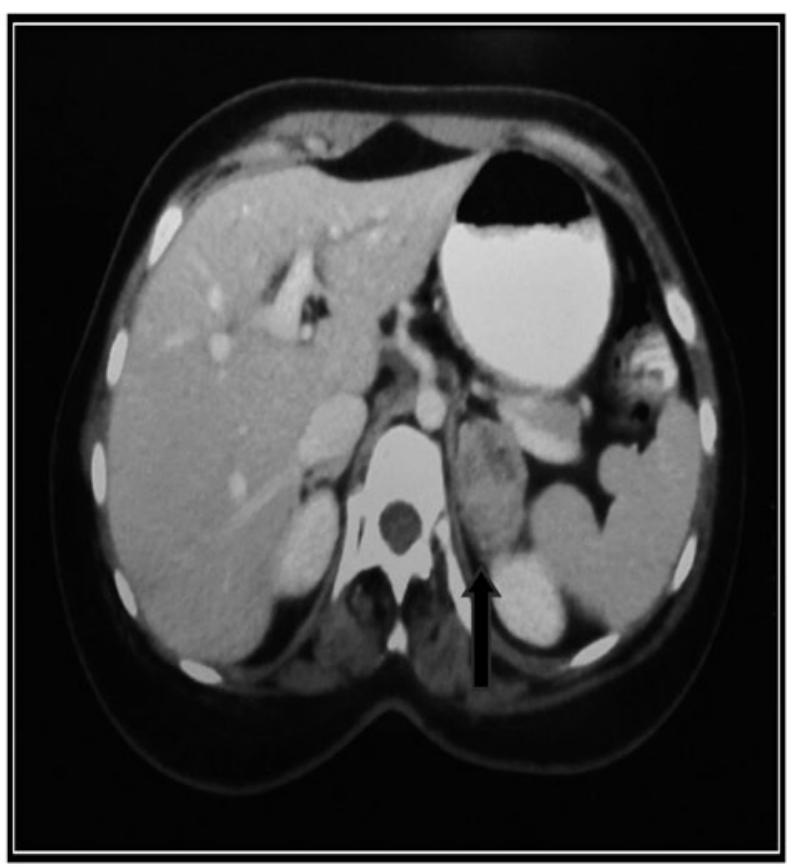

fue también normal en varias determinaciones. Tan sólo se objetivó una discreta elevación del acido homovanílico de 8,9 mg/24h (1-6,2 mg/24h). La cromogranina A era normal. Para completar el estudio de imagen se realizó una RMN abdominal donde se confirmaba la presencia de la masa adrenal izquierda de $3 \times 3,3 \times 5,5$ $\mathrm{cm}$, con señal heterogénea, realce tras administración de gadolinio y foco hipointenso en T1 e hiperintenso en T2 (Figura 2), que pudiera corresponderse a foco de necrosis o quística; todo ello en el contexto de la paciente pudiera corresponder a un feocromocitoma. Se solicitó una gammagrafía con meta-I-bencil-guanidina (MIBG) que no se realizó por incomparecencia de la paciente a la cita en primera instancia y posteriormente por falta del radionúclido, realizándose finalmente una gammagrafía con octreótido 111-In donde no se visualizaban depósitos patológicos del radiofármaco. Ante la sospecha de feocromocitoma no secretor la paciente es derivada al servicio de cirugía endocrina, que solicita la realización de angioRM de arterias renales, donde se confirmaba la presencia de la masa polilobulada en la glándula suprarrenal izquierda de 2,5 x 4,6 x 4,7 cm de comportamiento y señal heterogénea (hipointensa en T1 e hiperintensa en T2) con marcado realce tras administración de contraste, todo ello compatible con el diagnóstico de feocromocitoma. Se ingresó a la paciente para preparación prequirúrgica, realizándose, tras alfa y beta bloqueo, adrenalectomía laparoscópica izquierda sin complicaciones. En el estudio anatomopatológico de la pieza quirúrgica se identificó la presencia de un tumor constituido por 2 patrones: áreas predominantes de ganglioneuroma con células ganglionares sobre un estroma fascicular, y otras áreas menos extensas de feocromocitoma con células grandes, poligonales, con citoplasma granular y núcleo redondeado agrupadas en trabéculas, compatible con el diagnóstico de feocromocitoma compuesto. Tras la intervención quirúrgica, la paciente permanece asintomática, con tensiones arteriales normales, y niveles de catecolaminas, metanefrinas en
Figura 2. RMN: Masa adrenal izquierda con foco hiperintenso en T2 que puede corresponder a área de necrosis o quística

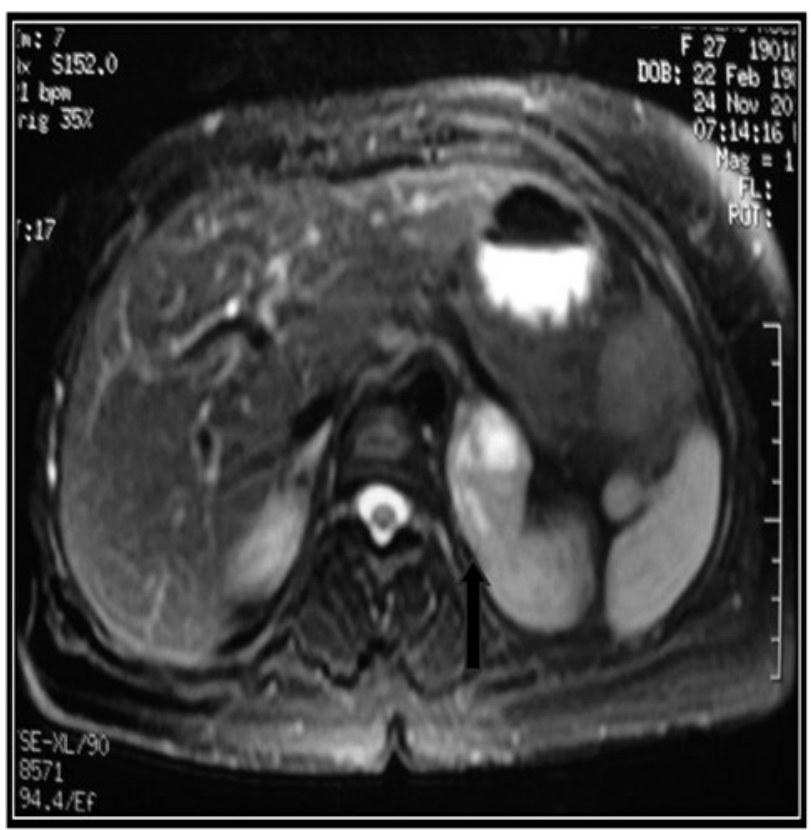

orina de 24 horas y cromogranina A también normales. En el TAC toracoabdominal no hay datos radiológicos de recidiva local ni de extensión a distancia.

\section{Discusión}

La NF-1 o enfermedad de Von Rechklinhausen es una enfermedad neurocutánea de herencia autosómica dominante. El diagnóstico es fundamentalmente clínico y se establece cuando están presentes al menos dos de los siguientes criterios (modificados en 1997 por la NIH) ${ }^{7}$ : 1) presencia de seis o más manchas café con leche con diámetro mayor de $5 \mathrm{~mm}$ en niños menores de seis años y mayor de $15 \mathrm{~mm}$ en adultos, 2) dos o más neurofibromas de cualquier tipo 0 un neurofibroma plexiforme, 3) efélides (pecas) en axila 0 ingles, 4) dos o más nódulos de Lisch (hamartomas del iris) , 5) glioma óptico, 6) displasia del hueso esfenoides o adelgazamiento de la corteza de los huesos largos con pseudoartrosis o sin ella y 7) familiar de primer grado con NF-1. La neurofibromatosis tipo 1 es unos de los síndromes que clásicamente se han asociado al feocromocitoma. Los feocromocitomas compuestos son tumores muy raros que también se han relacionado con este síndrome ${ }^{8}$. Asocian feocromocitomas con tumores de un mismo origen embrionario, que en más de un $70 \%$ son ganglioneuromas ${ }^{1}$. La mayoría se encuentran en las glándulas suprarrenales, aunque se han reseñado casos con localización extra-adrenal ${ }^{9}$. No hay predominio de sexo, y la mayor parte de los casos se han descrito en edades entre los 40 y 60 años ${ }^{1}$. Cuando dan síntomas son debidos a la secreción de catecolaminas ${ }^{10}$, aunque pueden presentarse de forma asintomática ${ }^{6}$. Los 
valores de catecolaminas y metanefrinas normales presentes en muchos pacientes pueden deberse a que el componente del ganglioneuroma modifica la secreción hormonal del feocromocitoma, por la metabolización de las catecolaminas ${ }^{2}$. Además, los niveles de metanefrinas y catecolaminas dependerán de la proporción entre ganglioneuroma y feocromocitoma, pues éste puede ser sólo un componente menor del tumor ${ }^{10}$. Suelen tener un comportamiento benigno, aunque se han descrito casos con metástasis a distancia ${ }^{11}$ y en algunos casos pueden ser más agresivos que los feocromocitomas clásicos, especialmente en los pacientes que se someten a una intervención quirúrgica o se encuentran bajo otras situaciones de estrés. El tratamiento no difiere del de cualquier feocromocitoma ${ }^{1}$, siendo el tratamiento quirúrgico por vía laparoscópica el de elección. Las directrices actuales para el manejo de pacientes con NF-1 recomiendan la realización anual de una historia clínica y exploración física general, considerando la mayoría de los autores esta práctica suficiente en la evaluación de los pacientes asintomáticos con NF-16. Sin embargo, la prevalencia de feocromocitoma y ganglioneuroma puede estar aumentada en pacientes con NF-1 y se ha observado que esta asociación se puede relacionar con tumores más agresivos, habiéndose encontrado afectación metastásica en el $60 \%$ de pequeños grupos de estos pacientes ${ }^{12}$. Por ello algunos autores recalcan, debido a la mayor agresividad de estos tumores si no se diagnostican a tiempo, la importancia de la evaluación clínica anual con estudios bioquímicos y pruebas de imagen, especialmente en pacientes con NF-1 con hipertensión, que están planificando un embarazo o que van a someterse a procedimientos invasivos ${ }^{6}$.

\section{Bibliografía}

1. Khan AN, Solomon SS, Childress RD. Composite pheochromocytoma-ganglioneuroma: a rare experiment of nature. Endocr Pract. 2010;16:291-99.

2. Aiba M, Hirayama A, Ito Y, Fujimoto Y, Nakagami Y, Demura H, et al. Compound adrenal medullary tumor (pheochromocytoma and ganglioneuroma) and a cortical adenoma in the ipsilateral adrenal gland.A case report with enzyme histochemical and immunohistochemical studies. Am J Surg Pathol. 1988;12:559-66.

3. Brady S, Lechan RM, Schwaitzberg SD, Dayal Y, Ziar J, Tischler AS. Composite pheochromocytoma/ganglioneuroma of the adrenal gland associated with multiple endocrine neoplasia 2A: Case report with immunohistochemical analysis. Am J Surg Pathol. 1997;21:102-8

4. Nakagawara A, Ikeda K, Tsuneyoshi M, Daimaru Y, Enjoji M. Malignant pheochromocytoma with ganglioneuroblastoma elements in a patient with von Recklinghausen's disease. Cancer. 1985;55:2794-8.

5. Juarez D, Brown RW, Ostrowski M, Reardon MJ, Lechago J, Truong LD. Pheochromocytoma associated with neuroendocrine carcinoma. A new type of composite pheochromocytoma. Arch Pathol Lab Med. 1999;123:1274-9.

6. Mezitis SG, Geller M, Bocchieri E, Del Pizzo J, Merlin S. Association of pheochromocytoma and ganglioneuroma: Unusual finding in neurofibromatosis type 1. Endocr Pract. 2007;13:647-51.

7. DeBella K, Szudek J, Friedman JM. Use of the national institutes of health criteria for diagnosis of neurofibromatosis 1 in children. Pediatrics. 2000;105:608-14

8. Kimura N, Watanabe T, Fukase M, Wakita A, Noshiro T, Kimura I. Neurofibromin and NF1 gene analysis in composite pheochromocytoma and tumors associated with von Recklinghausen's disease. Mod Pathol. 2002; 15:183-88.

9. Hu J, Wu J, Cai L, Jiang L, Lang Z, Qu G, Liu H, Yao W, Yu G. Retroperitoneal composite pheochromocytoma-ganglioneuroma: a case report and review of literature. Diagn Pathol. 2013; 8:63-7

10. Menon S, Mahajan P, Desai SB. Composite adrenal medullary tumor: A rare cause of hypertension in a young male. Urol Ann. 2011; 3:36-8.

11. Lam KY, Lo CY. Composite pheochromocytoma-ganglioneuroma of the adrenal gland: An uncommon entity with distinctive clinicopathologic features. Endocr Pathol. 1999;10:343-52.

12. Walther MM, Herring J, Enquist E, Keiser HR, Linbehan WM. Von Reckinghausen's disease and pheochromocytomas. J Urol. 1999;162:1582-86 